\title{
Crisis management during anaesthesia: recovering from a crisis
}

\author{
A K Bacon, R W Morris, W B Runciman, M Currie
}

Qual Saf Health Care 2005;14:e25 (http://www.qshc.com/cgi/content/full/14/3/e25). doi: 10.1136/qshc.2002.004333

See end of article for authors' affiliations .....................

Correspondence to: Professor W B Runciman, President, Australian Patient Safety Foundation GPO Box 400, Adelaide, South Australia, 5001, Australia; research@apsf. net.au

Accepted 12 January 2005
Preventing harm to the patient is the priority during a crisis. After a major incident, and especially when a patient has been harmed, there are a number of matters to be addressed: the ongoing care of the patient; documentation of the incident; investigation of the root causes; completion of reports; interviews with the patient and/or the next of kin, together with apologies and expression of regret; updates and ongoing support for friends and relatives; a word of thanks to the staff involved for their assistance; formal debriefing of staff for quality assurance and possibly ongoing support and a separate debriefing for psychological purposes; ensuring that the recommendations of the root cause analysis are carried out; or, failing that, that the issues are logged on a risk register.

The extent and depth of the follow up protocol depends on what, if any, harm may have been done. This may constitute completion of an incident report; notification of an equipment failure to a federal regulatory authority; arranging consultations with a mental health professional to manage psychological sequelae (especially following an awareness episode); follow up during weeks of intensive care treatment; or, when a death has occurred, a full medico-legal and/or coronial set of procedures. A précis is appended in an action card format.
O ther articles in this set of papers on crisis management are aimed at managing anaesthetic crises. This article considers the point at which the acute management of the incident is almost complete and the patient has or has not survived. What happens next?

The key steps are to decide if the patient has suffered any harm-actual or potential, physical or mental-and whether there is potential for a recurrence due to management, personnel, or equipment problems. If the answer is "yes" to any of these, then an appropriate post-mishap protocol should be invoked. When the incident has involved equipment or medical supplies, even if there is no damage to the patient, the aim must be to prevent it happening again-in your department or anywhere elsewhere in the world. The Australian Council for Safety and Quality has commissioned a formal standard for "open disclosure" after any significant iatrogenic event. ${ }^{\prime}$

\section{PATIENT MENTAL HEALTH SEQUELAE}

Anxiety, phobias, and post-traumatic stress disorders are possible patient sequelae of any anaesthetic mishap. Anxiety can usually be dealt with by explanation of normal technical procedures, with particular reference to the mishap that was experienced. Honesty and reassurance are the principles to be followed during the post mishap interview-it is possible to say sorry without admitting liability. As with all mishaps, the information and explanation should be repeated at least twice, on separate occasions, to allow the patient to understand and remember what has been said. It is preferable for the patient to have a relative or friend of their choosing to sit with them during at least one of these consultations. The vocabulary and phrases used must be appropriate to the situation, and the patient should be given plenty of time to clarify issues in their own mind. The interview should close with brief documentation of the interview in the medical record, and a definite plan for future anaesthetics, together with discussion of the possible use of an anxiolytic agent before any procedures. A letter of information should be given to the patient as well as to the referring medical practitioners.

Phobias, exaggerated anxiety, and irrational fears require expert advice from a psychiatrist or a psychologist. This is not the area for an enthusiastic and optimistic anaesthetist to grapple with the unplumbed complexities of the mental processes of the patient. It is useful for the anaesthetist to be present to formally introduce the mental health expert to the patient, and to explain the background to the problem. Once the consultation is in the control of the colleague, it may be appropriate for the anaesthetist to leave the room and let the mental health consultant continue. By making the initial introduction in person the patient will feel cared for. It also shows a continuity of care, a high degree of professionalism on the part of the anaesthetist, and helps prevent impressions of "not caring", "remoteness", "never got in touch with us", and all the other phrases so commonly quoted by aggrieved patients after any mishap, mental or physical. If it is not practical for the anaesthetist to be present, then it may be appropriate for the patient to be present when a prearranged phone call is made to the mental health consultant to outline the problem as is understood at that point. This is a useful technique to improve rapport and understanding. Introducing the patient and, if appropriate, selected friends and relatives to a social worker for ongoing practical support and advice may also be very helpful.

\section{AWARENESS UNDER ANAESTHESIA}

Patient awareness problems need special handling to avoid long term psychiatric morbidity such as post-traumatic stress disorder. ${ }^{1-9}$ The post-mishap routine to be followed is to talk with the patient at the first available opportunity, bearing in mind that the mishap may not be reported for many days. If the incident comes to light at the end of the stay in the postanaesthesia recovery unit, then the anaesthetist must address the problem at that point: ask the patient what they experienced. The start of the conversation should be quite natural, inquiring about present comfort, nausea, and so on. This can lead to specific comments such as: "The nurse tells 
me that you were dreaming/awake/felt pain; can you tell me about it?" If they are unable or appear unwilling to say what happened, then carefully recount the procedure from the time of the preoperative consultation, the entry to the induction or operating room, placement of the intravenous access, monitoring, and so on. The patient should then be asked what they remember of going to sleep (or not as the case may be), and the conversation allowed to develop using only such prompts as will enable the communication to continue. Do not ask the usual anaesthetic orientated questions that only allow a "yes" or "no" answer. In the unfamiliar territory of mental health questions, it takes time to extract the information that is relevant to the mental stresses inflicted by an episode of awareness.

When the facts required have been assembled, and the patient has been able to talk about their feelings, the interview should turn to an explanation and education phase. If the reason for the awareness is known, then a diplomatic and rehearsed presentation of the facts is appropriate. The problem may have been one of hypotension necessitating cessation of volatile agents, a syringe swap at induction, or an event where there were no obvious changes in physical signs to alert to the possibility of awareness. Cautious phrasing should be worked out with an experienced colleague to reassure the patient that they were not dreaming, not hallucinating, not grossly abnormal in any respect, and to begin to explain why it occurred. ${ }^{10}{ }^{11}$ When this phase is complete, discuss ways of preventing this occurring in the future and, as before, give the patient a letter of information that can be carried with them to give to an anaesthetist in the future. All the measures outlined in "Patient Mental Health Sequelae" above should, of course, be taken.

\section{MINOR MISHAPS WITH PHYSICAL SEQUELAE}

A minor mishap can be defined as one that causes no lasting damage to the patient. ${ }^{12}$ Examples of this are dental damage (which nevertheless may be expensive to repair), phlebitis, many drug extravasations, or a skin reaction to adhesive tape. Remember that patients and/or relatives may not perceive the mishap as "minor"! In most circumstances however, a minor mishap may be dealt with by applying common sense; but even when it is handled with honesty and sensitivity, it can still end up in the courts. ${ }^{12}$

\section{Dental damage}

Dental damage deserves a separate mention to highlight the protocol that should be in place. The cost of repairing any damage should be covered by hospital risk management, offering the services of a dental surgeon on the staff of the hospital. This not only keeps the problem within the organisation but may limit costs. If the patient requests their own dentist, the hospital staff should document the damage and inform the patient's dental surgeon of the damage and hospital policy.

\section{Communication}

It is crucial to establish good lines of communication with the patient and these must extend to next of kin, hospital medical staff, nursing staff, administrators, referring doctors, and, if the situation escalates, then communication links must include the family of the anaesthetist involved, and legal personnel. ${ }^{13}$

The interview with the patient should be arranged to allow the possibility of at least two separate conversations. The first may occur in an informal manner at the patient's bedside with the anaesthetist leading the consultation. The topic may be introduced by a sentence such as "There is a need to clarify something that occurred during anaesthesia". The events surrounding the mishap are described in non-technical language, the anticipated outcome of the problem indicated, and suitable documentation organised. The conversation should be briefly précised in the history and signed and dated. If the person is a legal minor or appears unable to understand, then a consultation with the next of kin is appropriate. A second interview is preferable to ensure that the patient's perception of events matches that of the anaesthetist. The patient is less likely to forget the details if he or she is in possession of an anaesthetist's report documenting the problem and, where appropriate, offering strategies to avoid similar events. Examples of this are: drug idiosyncrasies, unanticipated intubation problems, ${ }^{14}$ or reaction to antiseptic skin preparation.

\section{MAJOR MISHAPS WITH PHYSICAL SEQUELAE}

A major mishap is one that has serious effects such as death or permanent injury. ${ }^{12}$ This is a grave situation, and must have the medical team's full attention. Failure to come to terms with this will lead to unnecessary problems of communication, possibly litigation, and eventually, a lasting regret that the protocol was not invoked in full.

\section{Immediate actions}

\section{Contact a mentor or colleague}

Summon a senior colleague or mentor to assist you as soon as possible. The tasks ahead are unfamiliar, with no opportunity for rehearsal and fine tuning. One person cannot complete the tasks alone-the presence of a colleague is a "must". If the anaesthetist is not experienced in breaking bad news-as in an ICU setting - then a suitably proficient colleague should be recruited to assist.

\section{If the patient is dead}

When the incident has ended in death, the body is transferred to the custody of the Coroner or similar stateappointed officer. A senior anaesthetist should examine the body to record the equipment left in place and document the placement of crucial items-for example, an endotracheal tube, a central line. All statements provided for the authorities, including depositions for the Coroner, should be in draft form, then edited after careful scrutiny by the colleague and possibly a lawyer. The documentation should record facts, not opinions, and the more high profile the event, the more reason for the Coroner's deposition to be completed by the most senior anaesthetist in attendance at the anaesthetic. During the draft stage mistakes may be made which, if not corrected during the editing stage, will be given unwarranted emphasis in court at a later date. ${ }^{12}$ However, this must not be at the cost of failing to reveal all relevant details.

Relatives should be encouraged to view the body if they wish; this is a start to the normal grieving process. Two hospital staff should accompany them to explain the function of any equipment left in situ, and to answer questions on Coronial protocol, funeral arrangements, and so on. The departure of the family from the hospital should be carefully thought out, with medical and nursing staff accompanying them to their vehicle, or, if appropriate, arranging for a hospital car to take them home. There is no more pathetic sight than a new widow walking alone down a hospital corridor with a plastic bag of her late husband's clothes.

\section{If the patient survives}

The patient who survives a major mishap requires appropriate care, possibly in an intensive care unit (ICU), sometimes at another hospital. The transfer is another hazard and the anaesthetist or an appropriately trained, experienced, and accredited colleague should accompany the patient with such 
other staff as necessary and ample resources. In the ICU, especially if the patient is not under the direct care of the anaesthetist, it is important to insist on a detailed handover. This allows an opportunity to summarise the facts, provide verbal background information, and, at the same time, the medical record can be checked for detail and accuracy. Do not alter, deface, or cover up with correction fluid any part of the medical record, or replace a section with fresh pages. If there is an error, draw a single thin line through the sentence then add a newly timed and dated postscript. The process of editing is an important part of the care which may help to avoid needless litigation. All staff should be encouraged to record only facts; opinions need to be clearly identified as such, if it is felt essential for the ongoing care of the patient for them to be recorded.

\section{Communication with relatives}

This task is also high on the priority list, and is one that makes many anaesthetists feel very uncomfortable. From first principles, try not to deliver bad news over the telephone. If it is unavoidable, format the conversation in the same style as for a face to face interview. In the context of a major mishap, it is preferable to use the phone to invite the relatives to meet you in the hospital.

\section{The formal interview}

The actual room in which the interview takes place should be appropriate for a confidential conversation, both in size and freedom from intrusive sounds. It should be equipped with comfortable furniture and a telephone for outgoing calls. The interview must be a team event. UNDER NO CIRCUMSTANCES SHOULD THE SURGEON CONDUCT THE INTERVIEW ALONE. Having colleagues present helps to promote disciplined speech and helps to stop the casual, unrehearsed phrase which is capable of dual meaning. The team that conducts the interview should be: the anaesthetist, the surgeon, and a nurse. There may also be a need for a chaplain, social worker, or interpreter. A junior anaesthetist must be supported by a senior colleague.

The team should plan the interview to include:

- who will perform the introductions

- who will take the lead as spokesman

- what is to be said-exact phrases and sentences

- what is not to be said

- who will follow up medical matters

- who will be the ongoing communicator in the future

- who will act as supporter for the relatives.

\section{Introductions}

In an ideal scenario the person to do the introductions should be someone who has met the relatives in the past. This may be the surgeon, or another person. The task is simply to perform the introductions, confirm identities, shake hands, and ensure that the anaesthetist especially shakes hands and makes eye contact.

\section{Starting the interview}

Macnab ${ }^{15}$ advises that the prearranged spokesperson starts the conversation by stating how sorry they are to be the bearer of bad news. A phrase such as: "I am so sorry to have to tell you the dreadful news that ...". This is followed at once with the worst information: "During the operation your husband suffered a heart stoppage and despite all we could do for him, he died. I am so sorry."

In this manner, the next of kin have been warned that bad news is coming, that there is regret that it is bad news, the news itself, a hint of the major effort to right the situation, and finally an attempt to express sharing of grief. This is not the time to say exactly what happened because precise information will be lost in the shock of the moment. Those in the room will remember how the news was given, and the body language and facial expressions of the team.

At this point it may be appropriate to leave the family with the designated supporter. The brief departure of most of the team allows time for the family to absorb the impact. The supporter remains to listen and comfort, not to amplify anything that has been said. The supporter may become aware of special needs during this brief pause, and these matters can be taken up at the appropriate moment. During this break the team should assess the family's needs, the communication and comprehension requirements, and rehearse the key phrases once again.

\section{The explanations}

The second phase of the interview commences with reintroduction of the team, together with any latecomers who may have arrived as a result of perceived needs. Next, a further expression of personal sorrow and other appropriate sentiments, and a re-statement of basic sentences used to break the news in the first part: "I am so sorry that your husband has died. We tried everything we could, but the heart would not beat properly". The conversation should then aim to establish mutual understanding of the need for the procedure and, possibly, appreciation of risk factors. For medical staff in such unfamiliar roles, this is most comfortably accomplished by adopting a medical historytaking format-beginning at the first occasion that the patient or family should have become aware of the need for a procedure. "What can you tell me about your husband's health-when do you think he was last quite well? What do you think his general health was like? Why did he go to his doctor originally?" Having established the baseline, the interview can build up to the time of the anaesthetic and resuscitation, allowing the relatives to understand the implications of the illness. It is important to use nontechnical terms and to ensure that the next of kin have plenty of time to think about the replies. At this point the spokesman can describe the events in the operating room using an overview portrayal, not detailed technicalities. The phrases used should have been agreed to by the team before entry to the room unless no agreement is forthcoming, in which case a phrase may be used such as "We are not sure exactly what caused the problem, but we did see that the blood pressure was dropping and heart beat became very irregular". It is crucial to convey to the relatives that a great effort was made, that many experts came to assist, and that the team shares the family's grief. It demonstrates care.

The interview should stress known facts, not opinions. In the early stages after a mishap it is most unusual for all the facts to be known. Opinions offered as facts may have to be withdrawn in the light of subsequent investigations. Although the family will want to know why, ensure that they understand that at this early time not all the information is at hand. A tentative working diagnosis may be offered together with a promise that they will be updated as more information comes to hand.

If there are several interpretations of the events do not give a firm reason for the mishap. "I don't know" is truthful: you do not know for sure. This is especially important when a surgeon may consider that the anaesthetist was slow restoring blood volume, but the anaesthetist is sure the surgeon was slow to stop bleeding. What did happen was that the blood pressure dropped-that is fact. Likewise never imply blame on the patient. To them, the patient was not a high risk clinical problem with self inflicted problems of 
obesity and smoking; he was their father or husband. We all have our blind spots.

If the patient has survived the initial mishap, the interview should move on to explain current status, treatment, and possible prognosis.

When the relatives visit the ICU they need simple explanations about the equipment being used and what to expect as they enter the unit. Do not overwhelm them with science; allow the family to absorb what they see and to ask questions. They should be introduced to the doctor and nurse with primary responsibility for the friend or relative who has suffered harm.

\section{Follow up arrangements}

The welfare of the family is the next task to be considered, one which may appropriately be handled by a chaplain, social worker, friend, or neighbour. Once again, remember to shake hands and give them a parting message, one that they can relay to others. The anaesthetist should be available to attend interviews in which updated clinical information is made available, both to the relatives and other clinical staff. The constant presence of the anaesthetist is rarely a cause for comment, but absence is frequently criticised by family members. The anaesthetist or intensivist may wish to leave their mobile phone number with a key relative, to answer anything which may be troubling them.

\section{The next patient}

Should the next case proceed as scheduled? There may be some equipment fault or drug batch problem. Consider the effect if the next patient also dies. Unless the team is absolutely sure it was not one of these causes, the next case should be transferred to a new operating room, probably using a new team to allow the first team time to complete all the details that need attention. All equipment and drugs should be isolated for later examination if there is any chance that these may be implicated, and consideration given to allowing the medical team time off from their operating room duties. It is good management practice to allow medical and nursing staff to take a break. Avoid the "show must go on" mentality.

\section{Administration}

The anaesthetist must inform the hospital administration as soon as possible, so that hospital risk management processes can be activated and factual statements can be issued if there is a media inquiry. ${ }^{16}$ The inevitable incident forms, reports, and other paperwork are best drafted out at this timeanother reason for the team to take a break from clinical duties.

\section{Ongoing care of the surviving patient}

The ongoing hospital care may require appropriate expertise from colleagues in other disciplines, and coordination of these opinions and therapies should be linked by the anaesthetist. The medical record should be checked regularly, and the anaesthetist must make at least a daily visit to the unit during which time he or she should be seen by the relatives to be making that visit. The criticisms often include comments about the anaesthetist not coming to the ICU or the wards. If necessary, specific meetings with the relatives should be arranged.

\section{Equipment and drugs}

After isolation of the equipment and drugs, if this is deemed necessary, a detailed examination by an appropriate expert should be arranged who follows the national standard of the Australian and New Zealand College of Anaesthetists $(\text { ANZCA })^{17}$ or manufacturer's protocol. Drugs used must be checked, including batch number and expiry date. Following this, the used syringes and drugs should be placed in a sealed container, and impounded in a locked fridge for possible toxicological studies. If there is an equipment fault notify the manufacturer and the Therapeutic Goods Administration (TGA) in Canberra within Australia, and/or the equivalent other national body outside Australia.

\section{The referring doctors and other colleagues}

The communication links need to extend to colleagues who were involved in the care of the patient before this event. These may include other specialities as well as the family doctor. Opening a channel of communication allows a two way flow of information which should be used wisely to repeat the facts of the case. The hospital rumour system will usually beat any email, and seeding it with accurate information prevents a host of untrue hypotheses from gaining credence.

\section{End-of-shift defusing sessions}

The events of the day will affect many different people: nursing and medical staff, orderlies, and even other departments. A compulsory defusing session at the end of the shift is an opportunity for a brief talk to recount the events, to thank those who helped, and to talk about personal feelings that may result from the mishap. This 10 minute process, often in a tea room, should end with two points: the arrangements that have been made for care of the family, and to issue a "take away" statement that can be used to reply to enquiries from other people at work or in the community.

\section{Critical incident stress debriefing}

Mitchell ${ }^{18}$ has reported on a routine of critical incident stress debriefing (CISD) which may be useful when there are intense feelings. Such a routine is especially useful if there have been several stressful events before the current one. Sessions should include all staff who are from the same discipline and department. Cross discipline debriefing does not work well, and in particular the medical staff should have their own CISD sessions. These sessions should be held on the second or third day after the event, and be led by an experienced mental health worker, assisted by suitable trained peer supporters.

The benefits of CISD include better mental health of the group, improved teamwork, reduced resignation rates of senior and junior staff, and improved productivity. ${ }^{18}$ Common symptoms reported without CISD are: mental image flashbacks, poor sleeping habits, morbid fears of being involved in a similar incident, inability to concentrate, infringements of standard work practices, excessive alcohol consumption, burnout, and post-traumatic stress disorder.

Although the CISD concept started in emergency service workers, the need to maintain control at all times, to be calm despite everything else happening, is remarkably similar to the needs found in anaesthetists. The fact that an incident has occurred may cause the individual anaesthetist to fear for career and financial matters. The feelings may include an intensely internalised vulnerability, and for CISD to work, total confidentiality has to be assured before the session. The education phase of the CISD session teaches the truth of vulnerability and reports the feelings of other peer groups in similar situations. However, CISD is not a universal panacea, and identification of individuals who need extra professional help is a priority. The organisation of a CISD session has been considered elsewhere, and the format is well established. ${ }^{19}$ Some authorities have not endorsed the CISD concept, but involvement should at least be offered. 


\section{ACTION CARD}

After the Crisis - The Post Mishap Protocol

1. Contact a mentor/senior colleague: to come and assist in the protocol.

2. The patient: should be informed as soon as possible if he or she has survived. The Coroner's office is responsible for the body of a dead patient; the surviving patient usually needs intensive care and the anaesthetist must be seen to be there and to be a part of the team.

3. The relatives: Need to be informed promptly and accurately of what has occurred. THE SURGEON MUST NEVER CONDUCT THE INTERVIEW ALONE. This interview should be unhurried, run as a team effort, and may be divided into two parts - first the bad news, and then after a pause, the facts as known. Start with the need for the operation, what the plan was for the anaesthetic and build the basic medical history of the patient. If an opinion is to be offered, make it clear that it is an informed guess, not fact, the autopsy may prove you wrong. Ongoing support and communication channels of a surviving patient and their friends and relatives should be established.

4. The next patient deserves a fresh team and equipment. The unthinkable sequel to an unexpected death is that the next patient also dies because of an undetected equipment fault or overstressed personnel.

5. The equipment and drugs used, if implicated, must be isolated for examination.

6. The medical staff involved need a debriefing session to state what they think occurred, to ventilate their feelings and to learn from the mishap. Critical Incident Stress Debriefing (CISD) may be appropriate after two or three days.

7. The nursing and paramedical staff should attend an end of shift defusing session to allow immediate reactions to be revealed, and a factual and concise "take away" statement to be given out in order that the hospital staff have information that is appropriate and accurate. The situation may also require a formal CISD. Special consideration should be given to any anaesthesia nurse involved.

8. Administrative details: Check the medical record for accuracy and completeness, prepare formal statements, hospital or departmental incident reports, AIMS and TGA reports. The ICU record should be examined frequently. Never alter or destroy the record, but you may annotate with a separate timed, dated and signed postscript.

9. The anaesthetist, as well as the anaesthetist's family, should be supported and assisted in the immediate post catastrophe period.

10. The courts: Copy, review and understand all relevant records. Notify medical defence and hospital administration. Prepare a personal statement of events for medical defence even if no court action follows.

\section{After the Crisis - Notes}

\section{IF THE PATIENT SURVIVES}

Talk to the patient at the earliest opportunity

Explain what has happened

Keep it simple and stick to the facts

Avoid guesswork

Do not admit liability but do say sorry

For incidents with risk of psychiatric trauma/awareness/disability/ pain, involve a psychiatrist/psychologist.

If it was a minor mishap (e.g. superficial corneal or dental damage) Arrange for the problem to be corrected as soon as possible at no expense to the patient.

\section{IF THE PATIENT DIES}

Contact a senior colleague who will: Advise you to stop working that day/night

Help you with the tasks ahead

Liaise with medical/hospital administration

Arrange cover of your duties

Make a factual account as soon as possible referring to the medical record

Do not alter or erase any part of the anaesthetic record

You may annotate the medical record with a separately timed, dated and signed postscript

Leave tubes, cannulae, drains, lines etc. in situ

If there is any doubt at all about what happened, isolate the theatre/ equipment/drugs for future examination by an appropriate person

who can document the checks performed

Contact the family personally as soon as possible

Arrange an interview in a suitable room

DO NOT let others (e.g. surgeon) conduct the interview alone

Give the bad news first; show empathy and say sorry

Explain the facts as known at the time

Do not offer opinions lightly

Make yourself available to the family (e.g. contact number)

Debrief the staff involved

Seek a good friend/family member for support

Contact the relevant Medical Defence Organization

Contact the Head of Department

These notes comprise a page of the

Crisis Management Manual23.

Figure 1 Action card. After the crisis - the post mishap protocol.

\section{A personal record of events}

After any significant mishap, or where the immediate reaction of the patient or relatives is unfriendly, a personal record of events is extremely useful in subsequent legal proceedings. It should be started with the heading "this report is being composed in anticipation of legal action" and the completed report should be sent with a covering letter to the medical or hospital defence organisation. The report should contain every conceivable detail: what you did the previous day, what information you had before the case that was not recorded in the history, who was present during the case (you will not remember in two years' time) what their tasks were, what your plan was, what you saw, what you did, why you did it. As with any important document, it should be drafted, edited, and reworked until every wayward phrase, misspelling, error of decimal point, or unit of measurement has been removed. This is a task where the colleague who originally came to assist can be of great help. Finally, the anaesthetist should print out the final draft, sign, date, and send it, together with photocopies of relevant sections of the medical record, to their medical defence lawyer.

The concentration needed to produce the report has another benefit. The anaesthetist is starting a personal debriefing process. The very reason that the colleague has to sit down and discuss the report becomes a useful starting point to assist in the maintenance of mental health. Lawyers 
may advise the anaesthetist not to discuss the matter in public for fear of creating another witness; the truth of the matter is that unless the anaesthetist can debrief to a colleague, future mental health may be affected.

\section{Mental health of anaesthetists}

The mental health of anaesthetists is a not a topic that gets much publicity in the medical press, but suicide is reputed to be more frequent among anaesthetists than most other specialities. ${ }^{20}$ Legal action and major mishaps appear to be associated with many suicides. The problem is one of perception: “... professional isolation, loss of self esteem, possible revelation of a chemical dependency problem, loss of control over events that culminated in the mishap, loss of career prospects, potential loss of income, media exposure, and loss of community standing may result in the greatest loss of all: suicide. In this single act the anaesthetist regains control over his or her destiny, a devastating permanent answer to a temporary problem." ${ }^{\prime 12}$

So how do we overcome this problem? Provision of mentors, acceptance by heads of departments that they must have protocols to cover mishaps, and that these must include debriefing and support, creation of a workplace milieu where support takes precedence over competitiveness and reflex judgement; and an appreciation by all anaesthetists that they should be professionals who can recognise, and respond to, need in a colleague.

Currently, in Australia, a network of anaesthetists has been evolved to perform this role, and in some North American hospitals a "buddy" system has been created to try and prevent the feelings of isolation that are such a burden to the anaesthetist. ${ }^{21}$ Peers at work should be prepared to make opening comments such as "Sorry to hear of your problem the other day"; this is far better than an awkward silence in the changing room. There is a need for the supportive process to extend to the spouse or next of kin of the anaesthetist. Good support on the home front, sympathy, and a common purpose will help the healing of mental scars. The initial contact with the spouse opens another avenue of help.

Signs that mental health is deteriorating are varied, and include a change of personal habits, unusual seeking of solitude, reluctance to take part in conversations, poor appetite, weight loss, and so on. Such behaviour may extend to a chemical dependency, characterised by irrational displays of temper, inexplicable sudden mood swings, narcotic seeking behaviour, absenteeism, wearing long sleeves in theatre, complaints of coldness, chronic sniffing, failure to maintain eye contact at important times, nursing staff suggestions of increased and inappropriate narcotic usage, and so on. ${ }^{22}$ If chemical dependency is suspected, it is crucial to involve a psychiatrist with great experience in dealing with colleagues with chemical dependency. This is no place for well meaning amateurs.

\section{The legal processes}

Many anaesthetists admit to a private dread of appearing in court. The possibility of intrusive television cameras, adverse publicity, premature media judgements, and professional isolation are commonly mentioned. The legal process has its own rules, and just as our world of the operating suite is potentially terrifying to the layman, in the court we are the outsiders. To achieve the desired outcome, preparation must be very thorough. Every sheet of the medical record must be known in detail, and conference with the lawyers should take priority over all other day to day activities. Personal dreads can be reduced by visiting the court before the day, and becoming immunised by non-threatening small doses of the unfamiliar environment. The Coronial process in Australia is significantly different from other Australian courts, and the rules of evidence are more relaxed. The family are given latitude to understand the cause of death, and thus to move on through their natural grieving process. The usual advice given by lawyers is to stand up, speak up, and shut up-never, ever be tempted to add an "off the cuff" comment. However, never agree with anything that you know not to be true and do not allow your silence to be thus interpreted.

\section{CONCLUSION}

The protocols to be followed after a mishap are complex and give little opportunity for rehearsal. A trusted colleague must assist the anaesthetist from the first possible moment, and that person is a key to completing the process in full. An appendix is included in an action card format to assist in covering all the areas of this protocol (fig l, left hand panel) while the right hand panel is a reproduction of the algorithm which appears in a Crisis Management Manual published by the Australian Patient Safety Foundation. ${ }^{23}$ Every anaesthetist is

\section{Key messages}

- This article, in contrast to the other papers in this series, considers the time when the acute management of the incident is almost complete and the patient has or has not survived or suffered harm.

- The protocols to be followed after an anaesthetic related mishap are complex, with little opportunity for rehearsal. A trusted colleague must assist the anaesthetist, from the first possible moment until the very end of all proceedings.

- Key steps are to decide: has the patient been harmedactual or potential, physical or mental? Is there a possible recurrence of the incident due to management, personnel, or equipment problems?

- For any incident involving equipment or medical supplies, prevention of a recurrence anywhere in the world must be the aim. A more immediate related decision is whether to proceed with the next case.

- Honesty and reassurance are the principles for any post-mishap interview. It is possible to say "sorry" without admitting liability (such a premature admission may prove to be completely unjust when the real causes of the mishap are uncovered). Include your plans for future prevention of a recurrence.

- Provide a letter of information to both the patient (or family) and to the referring medical practitioners.

- Where patient harm or death has resulted, under no circumstances should the surgeon conduct the interview alone.

- Awareness under anaesthesia and dental damage each require special post-mishap considerations and protocols.

- Do not overwrite, deface, or cover up any part of the medical record. Delete any errors with a single line and add a new timed, dated, and signed postscript.

- Make a personal record of the events.

- The subsequent mental health of involved anaesthetists and anaesthetic staff and the dread of legal proceedings impose important special follow up requirements.

- A protocol action card is provided in this paper. 


\section{Further reading}

- Bacon AK, Mason RL. Cleaning up afterwards and reducing the threat of litigation. Bailliere's Clinical Anaesthesiology 1993;7:485-508.

- Cheney FW. Anaesthesia and the law: the North American experience. $\mathrm{Br} J$ Anaesth 1987;59:891900.

- Gibbs JM. No fault compensation for personal injury in New Zealand: some implications for anaesthetists. Br J Anaesth 1987;59:865-69.

- Kubler-Ross E. On death and dying. New York: Macmillan, 1969.

- Roberts RC. Risk management: an ounce of prevention. Wis Med J 1989;88:23-4.

- Finch JD. Legal obligations and responsibilities of the medical practitioner. Br J Anaesth 1987;59:870-6.

vulnerable to the unexpected; we all need to appreciate the informal mutual support we can offer each other.

\section{ACKNOWLEDGEMENTS}

The authors would like to thank all the anaesthetists in Australia and New Zealand who contributed to the 4000 incident reports upon which this and the other 24 papers in the Crisis Management Series are based. The coordinators of the project also thank Liz Brown for preparing the draft of the original Crisis Management Manual; Loretta Smyth for typing; Monika Bullock, RN, for earlier coding and classifying of data; Dr Charles Bradfield for the electronic version of the algorithms; Dr Klee Benveniste for literature research; Drs Klee Benveniste, Michal Kluger, John Williamson, and Andrew Paix for editing and checking manuscripts.

\section{Authors' affiliations}

A K Bacon, Consultant Anaesthetist, St John of God Hospital, Berwick, Victoria, Australia

R W Morris, Director of Research and Development, Sydney Medical Simulation Centre, Royal North Shore Hospital, St Leonards, New South Wales, Australia

W B Runciman, Professor and Head, Department of Anaesthesia and Intensive Care, University of Adelaide and Royal Adelaide Hospital, Adelaide, South Australia, Australia

M Currie, Clinical Quality Coordinator, Goulburn Base Hospital, Goulburn; and Consultant in Clinical Quality, Southern Area Health Service, New South Wales, Australia
This study was coordinated by the Australian Patient Safety Foundation, GPO Box 400, Adelaide, South Australia, 5001, Australia.

\section{REFERENCES}

1 Australian Council for Safety and Quality in Health Care. Open Disclosure Standard: A National Standard for Open Communication in Public and Private Hospitals Following an Adverse Event in Health Care. Canberra: Australian Council for Safety and Quality in Health Care, 2003. Available from http://www.safetyandquality.org/articles/Publications/ OpenDisclosure web.pdf (accessed 15 December 2004).

2 Cobcroft MD, Forsdick C. Awareness under anaesthesia: the patients' point of view. Anaesth Intensive Care 1993;21:837-43.

3 Macleod AD, Maycock E. Awareness during anaesthesia and post traumatic stress disorder. Anaesth Intensive Care 1992;20:378-82.

4 Liu WH, Thorp TA, Graham SG, et al. Incidence of awareness with recall during general anaesthesia. Anaesthesia 1991;46:435-7.

5 Salomons TV, Osterman JE, Gagliese L, et al. Pain flashbacks in posttraumatic stress disorder. Clin J Pain 2004;20:83-7.

6 Spitellie PH, Holmes MA, Domino KB. Awareness during anaesthesia. Anesthesiol Clin North America 2002;20:555-70.

7 Lennmarken C, Bildfors K, Enlund G, et al. Victims of awareness. Acta Anaesthesiol Scand 2002;46:229-31.

8 Ho AM. 'Awareness' and 'recall' during emergence from general anaesthesia. Eur J Anaesthesiol 2001;18:623-5.

9 Ghoneim MM. Awareness during anesthesia. Anesthesiology 2000;92:597-602.

10 Aitkenhead AR. Awareness during anaesthesia: what should the patient be told? Anaesthesia 1990:45:351-2.

11 Moerman N, Bonke B, Oosting J. Awareness and recall during general anesthesia. Facts and feelings. Anesthesiology 1993;79:454-64.

12 Bacon AK. Major anaesthetic mishaps-handling the aftermath. Curr Anaesth Crit Care 1990;1:253-7.

13 Bacon AK. Death on the table. Some thoughts on how to handle an anaesthetic-related death. Anaesthesia 1989;44:245-8.

14 Atkins RF. Simple method of tracking patients with difficult or failed tracheal intubation. Anesthesiology 1995;83:1373-4.

15 Macnab F. After the catastrophe, handling the distressed and hostile family. Austral as Anaesth 1990:83-6.

16 Aitkenhead AR. Anaesthetic disasters: handling the aftermath. Anaesthesia 1997; 52:477-82.

17 Australian and New Zealand College of Anaesthetists. Recommendations on checking anaesthesia delivery systems. PS31: 2003. Available from www.anzca.edu.au/publications/profdocs/profstandards/ps31_2003.htm (accessed 6 October 2004).

18 Mitchell JT. Crisis worker stress and burnout. In: Mitchell JT, Resnik HL, eds. Emergency response to crisis. Maryland: RJ Brady Co, 1981.

19 Mitchell JT. When disaster strikes the critical incident stress debriefing process. J Emerg Med Services 1983;8:36-9.

20 McNamee R, Keen RI, Corkill CM. Morbidity and early retirement among anaesthetists and other specialists. Anaesthesia 1987;42:133-40.

21 Davies JM, Bacon AK. When things go wrong: Pt 2, Doing what is right for the psychological well being of the anesthesiologist. Anesthesiology Review, 1990;XVII, 4:50-3

22 Lecky JH, Aukburg SJ, Conahan TJ, et al. A departmental policy addressing chemical substance abuse. Anesthesiology 1986:65:414-17.

23 Australian Patient Safety Foundation. Crisis Management Manual: COVER $A B C D$ A SWIFT CHECK. Adelaide: Australian Patient Safety Foundation, 1996, 74 pp. Available at http://www.apsf.net.au/anaesthesia.htm (accessed 6 September 2004). 